\title{
AGATA modules as Compton polarimeters for the measurement of gamma-ray linear polarisation
}

\author{
B. Melon ${ }^{1,2, a}$, P. G. Bizzeti ${ }^{1,2}$, P. Sona ${ }^{1,2}$, C. Michelagnoli3,4, D. Bazzacco ${ }^{3,4}$, E. Farnea ${ }^{4}$, \\ A. M. Bizzeti-Sona ${ }^{2}$, G. de Angelis ${ }^{5}$, A. Gadea ${ }^{6}$, A. Gottardo ${ }^{5}$, S. Lunardi ${ }^{3,4}$, S. M. Lenzi $i^{3,4}$, \\ R. Menegazzo ${ }^{4}$, D. Mengoni ${ }^{4,8}$, A. Nannini ${ }^{2}$, D. R. Napoli ${ }^{5}$, A. Perego ${ }^{1,2}$, F. Recchia ${ }^{5}$, E. Sahin ${ }^{5,7}$, \\ J. J. Valiente-Dobon ${ }^{5}$, and C. A. Ur ${ }^{4}$ \\ ${ }^{1}$ Dipartimento di Fisica ed Astronomia, Università degli Studi di Firenze, Italy \\ ${ }^{2}$ INFN, Sezione di Firenze, Italy \\ ${ }^{3}$ Dipartimento di Fisica, Università degli Studi di Padova, Italy \\ ${ }^{4}$ INFN, Sezione di Padova, Italy \\ ${ }^{5}$ INFN, Laboratori Nazionali di Legnaro, Italy \\ ${ }^{6}$ IFIC, CSIC -University of Valentia, Spain \\ ${ }^{7}$ Now at Department of Physics, University of Oslo, Norway \\ ${ }^{8}$ University of the West Scotland, United Kingdom
}

\begin{abstract}
The ability of a cluster of three segmented Ge crystals ("AGATA module") acting as a Compton polarimeter to measure the linear polarization of gamma rays has been investigated at an energy close to $511 \mathrm{keV}$. Partially polarized gamma rays have been produced by Coulomb excitation of the first excited state of ${ }^{104} \mathrm{Pd}$ a and ${ }^{108} \mathrm{Pd}$.
\end{abstract}

New generation of large detector arrays of highly segmented germanium crystals are under construction in Europe [1] (AGATA) and US [2] (GRETA) for $\gamma$ spectroscopy. With the implementation of Pulse Shape Analysis (PSA) and gamma-ray tracking techniques, the time-ordered sequence of energy releases (hits) in the germanium medium can be reconstructed for each gamma ray. In particular, in the Compton scattering process, the azimuthal and polar scattering angles can be determined from the locations of the first and second hits. Given these quantities, the performance of a highly segmented HPGe detector used as a Compton polarimeter is expected to be superior to a conventional one.

Compton polarimetry exploits the dependence of the Klein Nishina cross section (see eq. 1) on the azimuthal scattering angle. We have investigated the capability of individual AGATA modules, composed of three segmented Ge counters, to measure the linear polarisation of $\gamma$-rays of energy close to $511 \mathrm{keV}$ through the azimuthal distribution of their first Compton scattering. To this aim, a beam of ${ }^{12} \mathrm{C}$ ions at an energy of $32 \mathrm{MeV}$, provided by the XTU Tandem accelerator of LNL, has been used to Coulomb excite (CE) the first excited $2^{+}$state in ${ }^{104} \mathrm{Pd}$ and ${ }^{108} \mathrm{Pd}$, which de-excite by emission of partially polarized $\gamma$-rays of $558 \mathrm{keV}$ and $434 \mathrm{keV}$, respectively. Two AGATA modules of the AGATA Demonstrator, for a total of six Ge counters, have been mounted at angles as close as possible to $90^{\circ}$ with respect to the beam direction, where the polarisation reaches its maximum value. In a separate measurement, a ${ }^{137} \mathrm{Cs}$ source has been used, in order to provide the relative efficiency

\footnotetext{
a e-mail: melon@fi.infn.it
} 


\section{EPJ Web of Conferences}

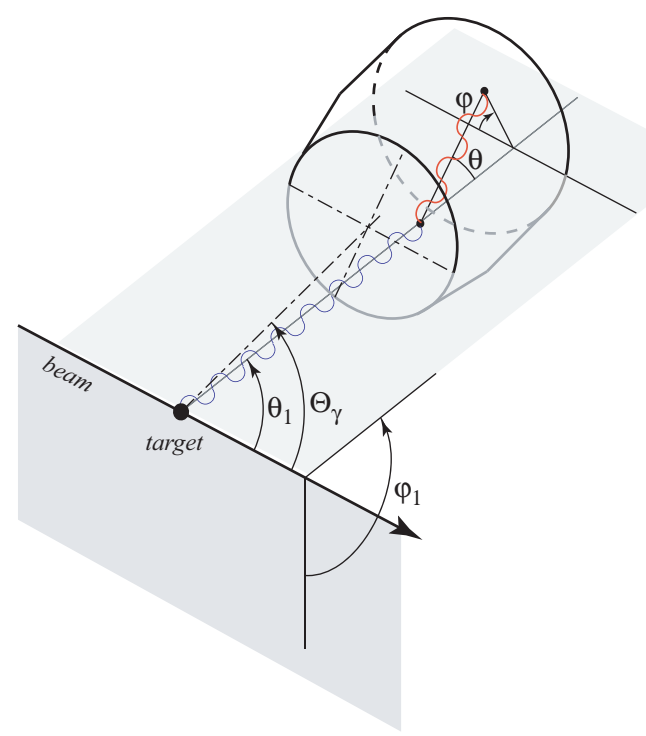

Figure 1. Schematic view of the geometry and kinematics of Compton scattering.

of the apparatus as a function of the azimuthal scattering angle. In Figure 1 a schematic view of the geometry and kinematics of Compton scattering is shown. The linear polarisation of a photon beam can be described in terms of Stokes parameters $\mathrm{P}_{1}$ and $\mathrm{P}_{2}$. For our specific case of photons emitted by Coulomb excited nuclei, these parameters are referred to the plane (xy) of a reference system (xyz) having the $\mathrm{z}$ axis along the gamma momentum and the $\mathrm{x}$ axis in the plane defined by the beam direction and the gamma momentum. The parameter $\mathrm{P}_{2}$ vanishes in our case due to the axial symmetry around the beam direction.

The theoretical expression for the Compton differential cross section for the case of the linear polarisation of a $\gamma$ population having Stokes parameters $P_{1}=P$ and $P_{2}=0$ can be expressed in the form

$$
\bar{\sigma}_{C}(\theta, \varphi)=\frac{r_{0}^{2}}{4}\left(\frac{E_{\gamma}^{\prime}}{E_{\gamma}}\right)^{2}\left[\frac{E_{\gamma}}{E_{\gamma}^{\prime}}+\frac{E_{\gamma}^{\prime}}{E_{\gamma}}-\sin ^{2} \theta(1+P \cos 2 \varphi)\right]
$$

where $\mathrm{r}_{0}$ is the classical electron radius and $\mathrm{E}_{\gamma}$ and $\mathrm{E}_{\gamma}^{\prime}$ are the energy of the primary and scattered photon, respectively. The expected polarisation of $\gamma$ rays following $\mathrm{CE}$ of ${ }^{104} \mathrm{Pd}$ and ${ }^{108} \mathrm{Pd}$ has been calculated by the means of the GOSIA code [3]. This code provides the full set of statistical tensors $\rho_{K, k}$ describing the initial alignment of the Coulomb excited nuclei referred to the beam direction. In our case, $\rho_{K, k}=0$ for $k \neq 0$ and the linear polarisation of the $\gamma$ rays emitted at a given angle $\Theta_{\gamma}$ (Fig. 1) is determined by the ratio of statistical tensors $\rho_{20} / \rho_{00}$ and $\rho_{40} / \rho_{00}$, related to the ratio of the population of the $m= \pm 2$ and $m= \pm 1$ sub-states to the population of the $m=0$ substate. As a first step, the data replay of the signals of the 36 segments and central contact of each Ge crystal has been performed following the standard AGATA procedure [4] up to the identification of the PSA hits. Such information has then been treated by the tracking algorithm, to establish, event by event, the time-ordered sequence of the hits.

To derive the polarisation information from Compton scattering events, several restrictions (cuts) have been imposed. We limited the values of $\cos \theta_{G}$, deduced from the position of the first and second 

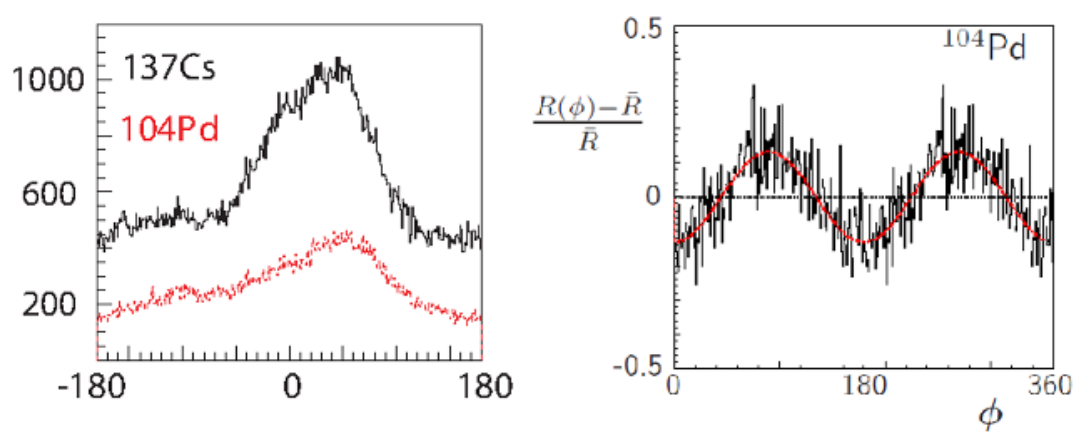

Figure 2. Examples of raw $\varphi$ distributions for gamma rays from ${ }^{137} \mathrm{Cs}$ and ${ }^{104} \mathrm{Pd}$ (left) and of the symmetrized distribution $R_{n}(\varphi)=(R(\varphi)-\bar{R}) / \bar{R}$ (right), where $\bar{R}$ is the average value of $R$, for gamma rays from CE of ${ }^{104} \mathrm{Pd}$. The continuous line corresponds to the fit of $R_{n}(\phi)$ by the function $A_{0}+A_{2} \cos 2 \varphi$.

hits (i.e. from the geometry), and $\cos \theta_{E}$, derived from the energy released to the electron in the first Compton scattering, to the interval $\left|\cos \theta_{G}-\cos \theta_{E}\right| \leq 0.1$. In order to reduce the uncertainty deriving from the experimental errors on the coordinates of the first and second hits, a cut on the distance $\mathrm{r}_{1,2}$ has been imposed, namely $r_{1,2} \geq 15 \mathrm{~mm}$. Finally, the scattering angles have been limited to $\left|\cos \theta_{G, E}\right| \leq 0.35$, in order to exclude the region where (for ${ }^{104} \mathrm{Pd}$ and ${ }^{137} \mathrm{Cs}$ ) an unresolvable ambiguity exists for the time-order of hits in the two-hit events [6].

Due to the complicate structure of AGATA modules, the detection efficiency is not constant with respect to the azimuthal angle $\varphi$. Indeed, in Fig. 2 (left panel) the $F(\varphi)$ distribution from an unpolarised source appears far from being flat. To correct for this effect we considered the ratio $\mathrm{R}(\varphi)$ of the $\varphi$ distribution of gammas from CE to the distribution from the ${ }^{137} \mathrm{Cs}$ source obtained by applying the same set of geometric and kinematic cuts. The ratio of the two distributions, $R(\varphi)=N_{C E}(\varphi) / N_{C s}(\varphi)$, shows indeed a kind of periodic dependence on $\cos 2 \varphi$.

Actually, due to the different energy of the primary gammas, the distribution of the polar angle $\theta$ and energy of the scattered photons (hence, their mean free path in the counter) from ${ }^{137} \mathrm{Cs}$ differs from those of CE $\gamma$ rays. Therefore, to correct for these effects, to each ${ }^{137} \mathrm{Cs}$ event is attributed a weight

$$
w(\theta)=\frac{\mu\left(E_{C E}^{\prime}\right) \exp \left(-\mu\left(E_{C E}^{\prime}\right) r_{12}\right) \sigma\left(E_{C E}, \theta\right)}{\mu\left(E_{C s}^{\prime}\right) \exp \left(-\mu\left(E_{C s}^{\prime}\right) r_{12}\right) \sigma\left(E_{C s}, \theta\right)}
$$

where $\sigma$ is the differential cross section for Compton scattering at the angle $\theta$ for the relevant energy of the primary gamma, $E^{\prime}$ is the corresponding energy of the secondary gamma and $\mu$ is the linear attenuation coefficient in germanium at the relevant energy of the secondary gamma.

In a simple Fourier analysis of the ratio $\mathrm{R}(\varphi)$, a small first term $(\propto \cos \varphi)$ appears in addition to the dominant term $(\propto \cos 2 \varphi)$. This can be attributed to the fact that edge effects do not compensate exactly, due to the asymmetric geometry of the AGATA cluster and also to the intensity variation of impinging gamma rays over the angular range spanned by a single counter. To correct for this effect, the $\mathrm{R}(\varphi)$ distribution for each counter has been symmetrized by substituting $\mathrm{R}(\varphi)$ and $\mathrm{R}(\varphi+\pi)$ with their average value. A typical result for the normalized quantity $R_{n}(\varphi)=(R(\varphi)-\bar{R}) / \bar{R}$ is shown in the right panel of Fig. 2. If $R_{n}(\varphi)$ is fitted with the function $A_{0}+A_{2} \cos 2 \varphi$, the ratio $A_{2} / A_{0}$ can 


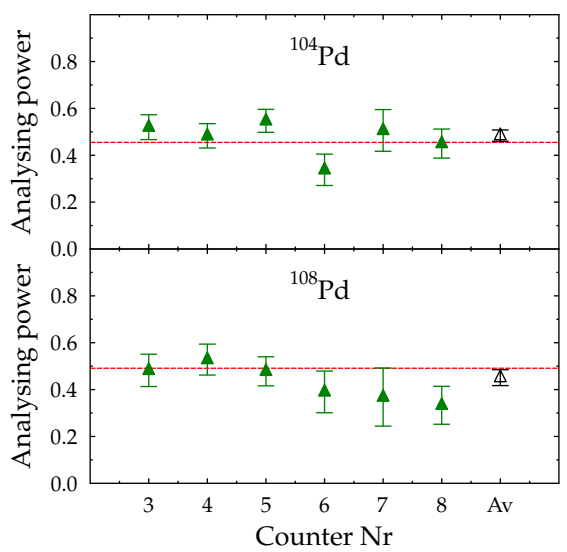

Figure 3. Analysing power $A_{s} / P$ of the six counters for the $555.8 \mathrm{keV}$ and $433.9 \mathrm{keV}$ gamma rays from CE of ${ }^{104} \mathrm{Pd}$ and ${ }^{108} \mathrm{Pd}$, respectively. The last point on the right is the weighted average for the whole set of counters (see text for details).

be interpreted as an asymmetry coefficient $A_{s}$ while the ratio of $A_{s}$ to $P$ (the calculated polarisation) gives the corresponding experimental value $\mathcal{A}$ of the analysing power [5]. The value of $\mathcal{A}$ for each counter is reported in Fig. 3; the last point on the right is the weighted average over the six counters. The horizontal line corresponds to the calculated values (practically independent of the counter). The latter have been obtained from the expression of the analysing power derived from the Klein Nishina formula corrected by a reduction factor accounting for the finite uncertainty of the coordinates of the first and second hits.

To summarize, the AGATA modules have been demonstrated to be an excellent tool for the measurement of the linear polarisation for gammas of energy close to $511 \mathrm{keV}$. This result led us to perform a second measurement with the AGATA clusters with the aim to determine to high precision the linear polarisation correlation of two entangled photons produced from the decay of the singlet state of positronium.

Two AGATA modules have been positioned symmetrically on opposite sides of a ${ }^{22} \mathrm{Na}$ source and coincidences between back-to-back $511 \mathrm{keV}$ gammas from positronium decay have been recorded. Data have been collected at three different distances from $\sim 3 \mathrm{~cm}$ up to $3000 \mathrm{~cm}$. For $\mathrm{N}$ recorded events, the linear polarisation correlation of the two entangled photons will be provided by the $\mathrm{N}$ true $\gamma-\gamma$ coincidences, while the reference distribution will be provided by the $\mathrm{N}(\mathrm{N}-1)$ non-correlated pairs (event mixing technique). The data analysis is in progress.

\section{References}

[1] S. Akkoyun et al., Nucl. Instr. and Meth. A 668, 26 (2012).

[2] M. A. Deleplanque et al., Nucl. Instr. and Meth. A 430, 292 (1999).

[3] T. Czosnyka, D. Cline, C. Y. Wu, GOSIA Manual, NSRL Report, University of Rochester 1983.

[4] D. Bazzacco, private communication.

[5] M. Suffert et al., Physica 25, 659 (1959).

[6] N. J. Hammond et al., Nucl. Instr. and Meth. A 547, 535 (2005). 